\title{
El contexto educativo como predictor de habilidades digitales y comportamientos riesgosos en Internet
}

\author{
Rolando Pérez SÁnchez* | David Torres FernándeZ***
}

El estudio busca determinar el efecto de la mediación tecnológica do-

Palabras clave

cente, la mediación de las amistades, el uso educativo de la Internet y las reglas asociadas al uso del teléfono celular en la institución educativa sobre las habilidades digitales y los comportamientos de riesgo. El estudio se llevó a cabo con 530 adolescentes costarricenses de entre 13 y 17 años, 52.6 por ciento mujeres y una edad promedio de 14.84 años $(D T=1.44)$. Se recurrió a un modelo de ecuación estructural, además de la utilización de análisis de mediación y moderación como procedimientos de análisis. Los resultados muestran que un mayor uso de la Internet en el colegio, una mayor mediación activa de los pares y niveles socioeconómicos más altos se asocian a mayores habilidades digitales, mientras que un mayor uso de la Internet se asocia a mayores comportamientos de riesgo, pero una mayor mediación activa de los pares se relaciona con un menor riesgo. Se discuten los resultados.

The present study seeks to determine the effect of teaching technological mediation, friendships mediation, educational use of the Internet and cell phone usage rules in educational institutions on digital skills and risk behaviors. In order to do this, we surveyed 530 Costa Rican teenagers between the ages of 13 and 17 -an average age of 14.84 years old $(S D=1.44)$-from which 52.6 percent were women. Furthermore, we applied a structural equation model as well as mediation and moderation analysis. The results show that a greater use of the Internet at school, greater active peer mediation, and higher socioeconomic status tend to result in higher digital skills; while a greater use of the Internet is associated with greater risk behaviors; also, a more active peer mediation tends to lower said risks.

Keywords

\section{Teaching}

Peer groups

Risks

Education and technology Teenagers

Recepción: 18 de mayo de 2020 | Aceptación: 15 de junio de 2021

DOI: https://doi.org/10.22201/iisue.24486167e.2021.174.59888

* Profesor catedrático de la Universidad de Costa Rica (Costa Rica). Doctor en Sociología. Línea de investigación: psicología social de los usos y efectos de las tecnologías digitales. Publicaciones recientes: (2021, en coautoría con C. Brenes, e I. Siles), "Predictores psicosociales de la exposición y difusión de noticias falsas en Costa Rica", Cuadernos. Info, vol. 49, pp. 214-238; (2020, en coautoría con D. Torres), "Mediación adulta de la Internet: un estudio cualitativo con adolescentes costarricenses", Revista Colombiana de Educación, vol. 79, núm. 1, pp. 39-60. CE: rolarez@ gmail.com

** Profesor asociado de la Universidad de Iberoamérica (Costa Rica). Licenciado. Línea de investigación: psicología social de los usos y efectos de las tecnologías digitales. Publicaciones: (2021, en coautoría con E. Blanca y R. Pérez (2021), "Inmersión y activación de estados emocionales con videojuegos de realidad virtual", Revista de Psicología, vol. 39, núm. 2, pp. 531-551; (2020, en coautoría con R. Pérez (2020), "Mediación adulta de la Internet: un estudio cualitativo con adolescentes costarricenses”, Revista Colombiana de Educación, vol. 79, núm. 1, pp. 39-60. CE: datofez1@yahoo.com 


\section{INTRODUCCIÓN}

Este estudio busca identificar los predictores de las habilidades digitales y los riesgos del uso de Internet en adolescentes, asociados al contexto escolar; específicamente, el caso de la mediación tecnológica docente, la mediación de las y los compañeros, el uso de Internet con objetivos educativos y las reglas asociadas al uso del teléfono celular en la institución educativa costarricense.

La institución escolar es un espacio fundamental en la vida adolescente, ya que ofrece espacios esenciales para la integración social, el aprendizaje y la formación de la identidad; en ella, docentes y pares ocupan una posición central (Eccles y Roeser, 2011), por lo que conocer el rol que juega esta institución en la promoción de habilidades digitales y la reducción de riesgo resulta relevante para la propuesta de medidas concretas para intervenir en estos ámbitos.

Se ha encontrado a nivel internacional una asociación entre habilidades digitales y la exposición a riesgos, misma que se ha explicado precisamente como producto de las posibilidades que ofrece el dominio del uso de la Internet para la exploración (Livingstone y Helsper, 2010; Livingstone et al., 2017; Livingstone et al., 2018; Rodríguez de Dios et al., 2018; Pérez Sánchez, 2019). Atendiendo a estos resultados, se considera necesario determinar cómo modificar esta asociación a partir del estudio de predictores relevantes para su comprensión.

Se entiende por habilidades digitales a la capacidad que tienen las personas para hacer uso de la Internet y las aplicaciones relacionadas. Se trata de capacidades que están supeditadas a las posibilidades de alfabetización digital con las que cuenten las personas, tanto en el contexto familiar, del grupo de pares o de la educación formal (Van Deursen y Van Dijk, 2014). Como señalan Van Deursen et al. (2014) se trata de capacidades asociadas a dos conjuntos básicos de habilidades: por un lado, al uso del medio, es decir, los dispositivos y aplicaciones, así como al manejo y configuración del navegador; y, por otro lado, las capacidades vinculadas al uso, procesamiento y comprensión del contenido, referidos a la selección y apropiación de información y la comunicación.

A partir de lo anterior se pueden identificar habilidades específicas: operacionales (saber cómo usar el navegador, cómo configurarlo para resguardar la seguridad en línea o cómo configurar las aplicaciones en uso); informacionales (la capacidad para buscar información, distinguir información confiable de la que puede ser falsa); sociales (la habilidad para comunicarse con otras personas de forma adecuada según la plataforma o aplicación, y conocer la configuración de la privacidad en redes sociales); y las habilidades para la creación de contenido (capacidad para desarrollar contenido, ya sea aplicación, contenido informativo o audio-visual y la posibilidad de compartirlo) (Helsper y Van Deursen, 2018).

Mientras tanto, por riesgo se entiende la probabilidad de ser dañado (Livingstone et al., 2018). El riesgo no es algo extrínseco a la actividad de las personas en sociedad; por el contrario, es intrínseco, se genera en el contexto de la misma sociedad y es resultado de la actividad digital, una parte fundamental e inseparable de las sociedades modernas (Livingstone, 2013). Por sí mismo, el riesgo no implica daño; debe considerarse y evaluarse el contexto protector de la familia, escuela o amistades, así como las habilidades digitales para identificar, prevenir o evitar las situaciones de riesgo. Los riesgos pueden clasificarse dependiendo de si la persona es receptora, participante o actor en la actividad riesgosa (Livingstone et al., 2015). En el caso de que la persona sea receptora, el foco de atención se encuentra en el tipo de contenido que recibe; cuando la persona es participante, el énfasis se pone en el tipo de actos respecto de los cuales resulta víctima; mientras que, cuando el foco de atención está en la persona como agente activa, se trata de identificar el tipo de actos violentos perpetrados por ella. 
Específicamente para este estudio se consideraron como dimensiones de riesgo el uso problemático del celular, el contacto en línea con personas desconocidas, recibir y enviar imágenes de contenido sexual, y haber sido víctima, presenciado o haber perpetrado ciber-matonismo. En estas actividades de riesgo, el o la adolescente puede ser actor, receptor (víctima) o participante.

Sobre el papel de la institución educativa en la promoción de habilidades digitales y la prevención o la exposición a riesgos, Berger y Wolling (2019), Kalmus y Roosalu (2012) y Lorenz et al. (2016) indican que el desarrollo de habilidades digitales protectoras en las y los adolescentes se favorece en instituciones que promueven la integración de las tecnologías de la información y la comunicación y que facilitan que los docentes las integren en sus actividades académicas.

Ahora bien, una de las tecnologías cuya introducción ha resultado más debatible en el campo educativo es el teléfono celular (O’Bannon et al., 2017), que en Costa Rica es el principal medio de acceso a Internet (Pérez Sánchez, 2019) y con ello la principal herramienta, tanto para el desarrollo de habilidades digitales, como para la prevención del riesgo. Al respecto, la discusión se ha centrado tanto en su uso en contexto de clase, como en establecer reglas claras en el caso de que se permita su uso educativo. Esto se relaciona con la creencia de que son los maestros quienes tienen la responsabilidad de enseñar sobre los usos seguros de la Internet, aun cuando el conocimiento que se tiene sobre la mediación docente sigue siendo escaso (Wishart, 2004; Soo et al., 2015; Bartau-Rojas et al., 2018).

Al respecto, Tingir et al. (2017) y Sung et al. (2016), en un estudio meta-analítico, reportan resultados positivos sobre el rendimiento al usar de forma sistemática el teléfono celular como parte de las actividades pedagógicas en clase; mientras que Kates et al. (2018) se muestran más cautelosos en identificar efectos positivos o negativos a partir de diversas investigaciones previas. Estos resultados llevan a incluir, como variable a indagar en el presente estudio, la introducción de reglas claras para el uso de teléfono celular por parte de la institución educativa, tanto para estimar el posible efecto sobre las habilidades digitales como para reducir la exposición y comportamientos de riesgo.

Otro aspecto que se ha observado es que enseñar a usar la Internet cambia las conductas de enseñanza y estos cambios dependerán de las características individuales de los docentes (habilidades digitales, capacidad de aprender por sí solos y actitudes hacia la tecnología), así como de las características contextuales (temas de hogar, reglas en la escuela, interacciones con amigos) y el bagaje de aprendizaje sobre tecnologías de la información que hayan tenido en su formación. De esta manera, escuelas con políticas más abiertas y preparadas para la enseñanza de Internet, así como docentes con mayor bagaje y mejores actitudes hacia esta red tendrán conductas de enseñanza más positivas en cuanto a la preparación de clases y el uso de materiales y evaluaciones en línea (Pimdee y Leekitchwatana, 2019).

Buena parte de la investigación sobre mediación de las tecnologías digitales, es decir, formas de instrucción, acompañamiento, monitoreo o restricción en el uso de la Internet, se ha focalizado en la mediación parental, y se han estudiado diferentes formas de mediación. En el caso de la presente investigación interesa estudiar, específicamente, la mediación activa, es decir, la orientada al acompañamiento y guía en el uso de la Internet por parte de pares y docentes. A diferencia de la mediación activa parental, la mediación activa de las y los pares se orienta más a acompañar y aconsejar en las mejores formas de uso de la Internet que a instruir u ofrecer apreciaciones críticas sobre el uso de esta tecnología (Livingstone et al., 2011; Shin y Lwin, 2017). En la adolescencia, el significado social, comunicativo y emocional de las amistades o compañeros de escuela es fundamental, por lo 
que la información que se recibe de ellos será significativa. Por su lado, la mediación activa docente se orienta a guiar y aconsejar en el uso de páginas que promuevan los aprendizajes en la escuela o que aporten información relevante para la vida cotidiana de las y los adolescentes, además de informar sobre el uso seguro y la reducción del riesgo (Shin y Lwin, 2017). En el periodo adolescente la orientación de las y los docentes es relevante, ya que aporta conocimientos y experiencia adulta que las madres o padres no proporcionan.

En el caso de la mediación de las amistades, la investigación se ha enfocado en la influencia o el apego, más que en la mediación activa, y se han encontrado casos de asociaciones positivas entre el apego y conductas de riesgo (Soh et al., 2018), así como un efecto de moderación entre la afiliación a pares problemáticos y la relación entre la conexión con la escuela y el uso problemático del Internet (Li et al., 2013).

Los estudios que se han enfocado propiamente en la mediación activa de las amistades muestran que las y los pares ejercen influencia en el ingreso y el uso de las redes sociales, así como en la iniciación en diferentes actividades en Internet (Barbovschi et al., 2015); esto podría permitir afrontar posibles riesgos, ya que este tipo de mediación facilita la confianza y la tematización sobre posibles amenazas (Cerna et al., 2016).

Con respecto a la mediación activa docente, se ha encontrado que ésta contribuye a disminuir los posibles riesgos respecto del acceso a Internet, al advertir sobre posibles daños y guiar su uso en un marco de confianza (Shin y Lwin, 2017). De igual manera señalan que la mediación docente disminuye los efectos negativos de la ciber-victimización y reduce la adicción a la Internet, a la cual se ha asociado este tipo de violencia (Jia et al., 2018). Berger y Wolling (2019) señalan que las y los docentes con formación para el uso de tecnologías digitales, unido a una institución educativa equipada para promover actividades que recurren a la Internet promueven el desarrollo de habilidades digitales asociadas a la protección. Kalmus et al. (2012), encontraron una asociación positiva entre la mediación docente y el desarrollo de habilidades digitales. Livingstone et al. (2011) identificaron que, en estratos socioeconómicos bajos, y en adolescentes de mayor edad, las y los docentes son la principal fuente de mediación tecnológica. Además, se ha encontrado que la manera como los docentes integran las tecnologías de la información y comunicación en la enseñanza puede influir en el uso desmedido de la Internet por parte de los menores de edad (Hsu, 2015).

Inclusive, se ha encontrado que desarrollar programas relativos al uso de Internet en estudiantes de secundaria puede influir de forma positiva en los estilos de vida de los y las jóvenes, y ayudarles a mejorar su dieta, patrones de sueño, ejercicio físico y uso de Internet, así como pasar de usos moderados a usos más ligeros de la red (Tekeste Fiseha y Razon-Estrada, 2018).

Las brechas digitales tradicionales asociadas al nivel socioeconómico definen el uso en el contexto costarricense. Pérez Sánchez (2019) encontró un mayor uso de Internet en los niveles socioeconómicos más altos, así como la presencia tanto de mayores habilidades digitales como de riesgos. Estos resultados hacen relevante identificar si las diferencias socioeconómicas hacen que el contexto educativo permita o no la promoción de habilidades digitales y la protección de situaciones de riesgo asociadas al uso de Internet. Por esta razón se propone el modelo que se muestra en la Fig. 1.

Con base en las consideraciones conceptuales y la investigación antecedente se proponen las siguientes hipótesis:

- H1 Una mayor mediación activa por parte de los docentes se asocia al desarrollo de mayores habilidades digitales.

- H2 Una mayor mediación activa por parte de las amistades se asocia al desarrollo de mayores habilidades digitales. 
- H3 Una mayor mediación activa por parte de los docentes se asocia con una reducción del riesgo.

- H4 Una mayor mediación activa por parte de las amistades se asocia con una reducción del riesgo.

- H5 Instituciones educativas con reglas claras sobre el uso del teléfono celular se asocia a una reducción de los riesgos.

- H6 Instituciones educativas con reglas claras sobre el uso del teléfono celular se asocia a una reducción en el desarrollo de habilidades digitales.
- H7 Un mayor uso de Internet para actividades educativas se asocia con mayores habilidades digitales.

- H8 Un mayor uso de Internet para actividades educativas se asocia con mayores riesgos.

- H9 La condición socioeconómica más alta presentará mayores habilidades digitales.

- H10 La condición socioeconómica más alta presentará mayores riesgos.

- H11 Existe una covariación entre las habilidades digitales y las actividades asociadas a riesgo.

Figura 1. Predictores asociados al contexto escolar de las habilidades digitales y la exposición a riesgos

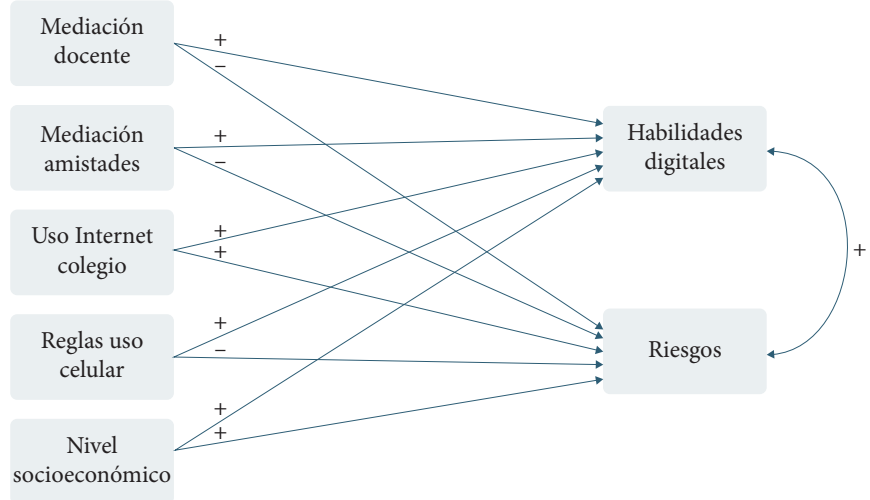

Fuente: elaboración propia.

De igual manera se considera que el contexto educativo puede ser un facilitador o inhibidor de las relaciones entre el tipo de mediación activa del o la docente o las amistades y las habilidades digitales o la presencia de riesgos. De allí que se propone poner a prueba el efecto mediador de la frecuencia de uso de la Internet en el colegio sobre la relación entre el tipo de mediación y su efecto sobre las habilidades digitales o los riesgos, así como el efecto moderador de la presencia de reglas en la institución educativa para el uso del teléfono celular (en tanto es el dispositivo más empleado para acceder a Internet) sobre esta relación entre los tipos de mediación y las habilidades o riesgos. En este sentido, se proponen las siguientes hipótesis (Figs. 2 y 3):

- H12 La asociación entre la mediación docente y las habilidades digitales está mediada por la frecuencia de uso de la Internet en el colegio.

- H13 La asociación entre la mediación docente y los riesgos en Internet está mediada por la frecuencia de uso de la red en el colegio.

- H14 La asociación entre la mediación de las amistades y las habilidades digitales está mediada por la frecuencia de uso de la Internet en el colegio. 
Figura 2. Efecto mediador hipotetizado de la frecuencia de uso de la Internet en el colegio sobre la asociación entre los tipos de mediación estudiado y las habilidades digitales y la presencia de riesgos
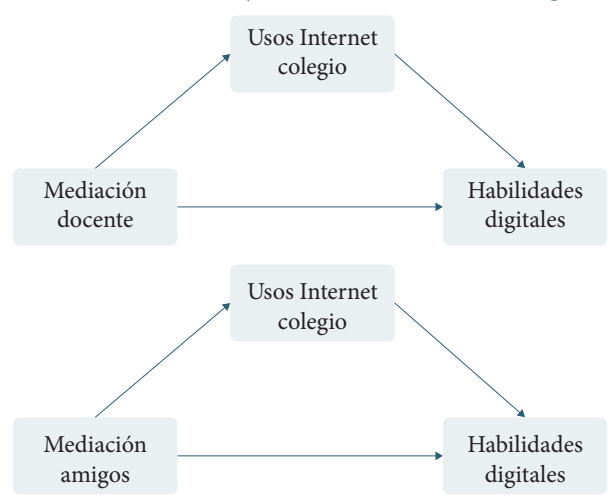

Fuente: elaboración propia.

- H15 La asociación entre la mediación de las amistades y los riesgos está mediada por la frecuencia de uso de la Internet en el colegio.

- H16 La asociación entre la mediación docente y las habilidades digitales está moderada por la presencia de reglas de uso del teléfono celular en el colegio.

- H17 La asociación entre la mediación docente y los riesgos en Internet está moderada por la presencia de reglas de uso del teléfono celular en el colegio.

- H18 La asociación entre la mediación de las amistades y las habilidades digitales

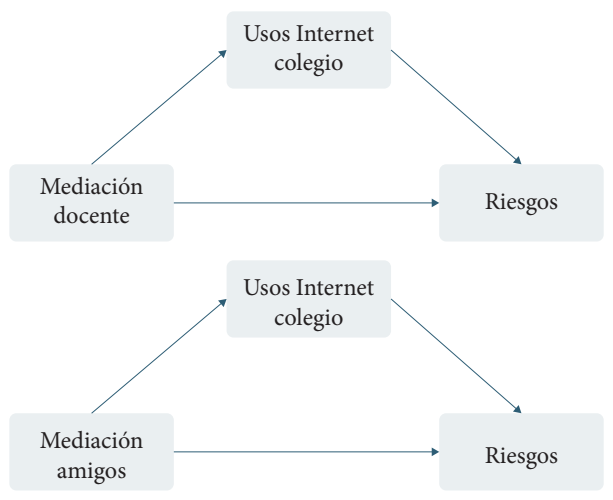

está moderada por la presencia de reglas de uso del teléfono celular en el colegio.

- H19 La asociación entre la mediación de las amistades y los riesgos está moderada por la presencia de reglas de uso del teléfono celular en el colegio.

\section{MÉTODO}

Esta investigación se inscribe dentro de la encuesta nacional Kids Online, representativa a 1 mil 008 niñas, niños y adolescentes entre 9 y 17 años, la cual está asociada a la red internacional Global Kids Online de UNICEF.

Figura 3. Efecto moderador hipotetizado de la presencia de reglas para el uso del teléfono celular en el colegio sobre la asociación entre los tipos de mediación estudiado y las habilidades digitales y la presencia de riesgos
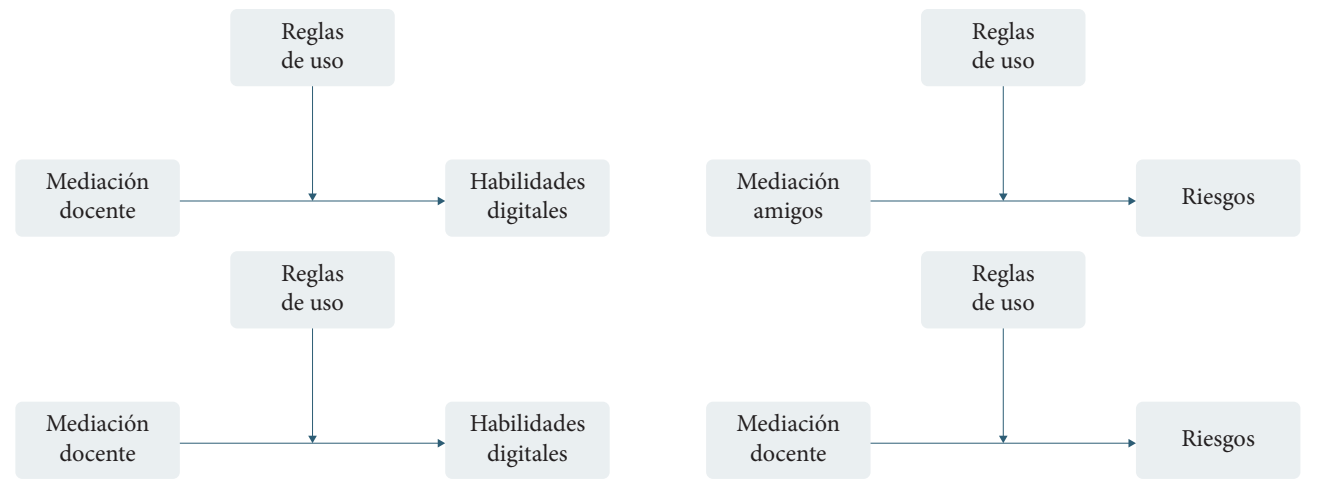

Fuente: elaboración propia. 


\section{Participantes}

El estudio utilizó un muestreo aleatorio, estratificado, polietápico, considerando como criterio de estratificación la región, urbana o rural. Para la recolección en las unidades muestrales encuestadas se controló la distribución por edad y género. La muestra presenta un error máximo de 3 por ciento y un nivel de confianza de 95 por ciento.

Los datos sobre los cuales se realizaron los análisis toman la submuestra de adolescentes entre 13 y 17 años, para un total de 530 adolescentes $(M=14.84, D T=1.44), 67$ por ciento de zonas urbanas y 52.6 por ciento mujeres.

La encuesta se efectuó en los hogares de las personas adolescentes. Previamente se solicitó el consentimiento del adulto al cuidado del/de la adolescente.

\section{Instrumentos}

Las mediciones empleadas corresponden al instrumento de la encuesta Global Kids Online, que retoma específicamente la versión del cuestionario empleado en Chile.

- Mediación activa docente: el instrumento toma como base la medida empleada en EU Kids Online (Global Kids Online, 2021). Se dirige a evaluar actividades realizadas por las y los docentes orientadas a la guía, acompañamiento y recomendación en el uso de la Internet. La medida consta de 12 ítems, y recurre a una escala de respuesta de 5 puntos, de nunca a siempre. Ejemplos de ítems son, "el profesor ayuda a usar Internet de forma segura", "el profesor explica que algunas páginas web son mejores que otras". Se obtuvo un coeficiente alfa de Cronbach de .90 . Se trata de una medida unidimensional.

- Mediación activa amigos: también toma como base el cuestionario empleado en EU Kids Online (Global Kids Online, 2021). Se dirige a evaluar comportamientos de las y los pares dirigidos a la guía y recomendación en el uso de la Internet. La medida consta de 4 ítems, en una escala de respuesta de 5 puntos, de nunca a siempre. Un ejemplo de ítem es el siguiente: "los amigos aconsejan cómo usar Internet de forma segura". La medida es unidimensional, presenta un coeficiente alfa de Cronbach de 84 .

- Habilidades digitales: es una versión reducida del cuestionario desarrollado por Helsper et al. (2015). Se dirige a evaluar las capacidades que tienen las y los adolescentes para hacer uso de la Internet, tanto el navegador como las aplicaciones asociadas, considerando aspectos como manejo de la información, privacidad, comunicación o creación de contenido. La medida es unidimensional. El instrumento está conformado por 10 ítems, con una escala de respuesta de 4 puntos, de "nada capaz" a "totalmente capaz". Este instrumento obtuvo un coeficiente alfa de Cronbach de 85 .

- Índice de riesgo: para esta investigación se construyó un índice de riesgo a partir de variables que se consideran básicas para entender el comportamiento y exposición a riesgo que pueda estar asociado eventualmente a daño. El índice es la sumatoria del siguiente conjunto de preguntas: uso excesivo del teléfono celular, contacto en línea con personas desconocidas, enviar o recibir imágenes con contenido sexual, reporte de haber sido víctima o perpetrado matonismo, para un total de 12 ítems, con respuestas dicotómicas.

- Regulación para el uso del teléfono celular en el colegio: se incluyó una variable dicotómica que indaga sobre la presencia de reglas para el uso del teléfono celular en la institución educativa.

- Índice de uso de la Internet en el contexto escolar: indaga acerca de la frecuencia con la que realiza diferentes actividades en el colegio con Internet. 
Por ejemplo, búsqueda de información, trabajo en grupos, preparación de tareas, elaboración de presentaciones, comunicarse con docentes. Consta de 8 ítems que utilizan una escala de 6 puntos, de nunca a todos los días. Se procedió a realizar una sumatoria de todos los ítems.

- Índice de nivel socioeconómico: sumatoria de un listado de bienes y servicios del que dispone la vivienda de las y los adolescentes encuestados, aunado a la pregunta de si posee su propia habitación.

\section{Procedimientos de recolección}

Inicialmente se procedió con la revisión y adaptación a los usos del lenguaje español de Costa Rica. Seguidamente se realizaron entrevistas cognitivas a 40 personas de la población meta de la encuesta, lo que permitió la realización de correcciones para la mejora en la comprensión de los ítems y después se efectuó una prueba piloto a 100 participantes, que de igual manera contribuyó a mejorar el proceso de recolección.

Para la aplicación definitiva se realizaron visitas a las casas de las y los adolescentes. Se entrenó previamente a las y los encuestadores. Se recurrió al uso de tabletas para recoger la información.

\section{Procedimientos de análisis}

Para el análisis de los datos se efectuaron análisis descriptivos, correlaciones bivariadas y la determinación de consistencia interna para las medidas estandarizadas. A continuación, se puso a prueba un modelo de ecuación estructural para identificar los predictores asociados al contexto educativo de las habilidades sociales y los riesgos posibles. Para este último análisis se consideró lo planteado por Hooper et al. (2008).

\section{Resultados}

Inicialmente se llevaron a cabo correlaciones bivariadas (Tabla I). Se estimó la asociación entre las habilidades digitales, los comportamientos de riesgo y la mediación activa docente y de pares, junto con el uso de la Internet en el contexto escolar, la presencia de reglas en el colegio para el uso del teléfono celular (que se introdujo como variable dummy) y el nivel socioeconómico.

Las habilidades digitales presentan una asociación positiva con la mediación docente $(r=.30)$ y la de pares $(r=.17)$, uso de Internet en el colegio $(r=.27)$, nivel socioeconómico $(r=.22)$, pero también con el índice de riesgo $(r=.23)$. Esto significa que mayores puntajes en las habilidades digitales se relaciona con

Tabla 1. Correlaciones bivariadas de las variables en estudio

\begin{tabular}{|c|c|c|c|c|c|c|c|c|}
\hline & M & DT & 1 & 2 & 3 & 4 & 5 & 6 \\
\hline 1. Habilidades digitales & 6.97 & 1.65 & - & & & & & \\
\hline 2. Índice de riesgo & 4.47 & 3.22 & $0.232^{* * *}$ & - & & & & \\
\hline $\begin{array}{l}\text { 3. Uso de la Internet en el } \\
\text { colegio }\end{array}$ & 3.09 & 2.13 & $0.273^{* * *}$ & $0.190^{* * *}$ & - & & & \\
\hline $\begin{array}{l}\text { 4. Mediación activa } \\
\text { docentes }\end{array}$ & 1.97 & 1.22 & $0.301^{* * *}$ & -0.089 & $0.240^{* * *}$ & - & & \\
\hline 5. Mediación activa pares & 8.00 & 5.39 & $0.173^{* *}$ & 0.022 & $0.230^{* * *}$ & $0.478^{* * *}$ & - & \\
\hline $\begin{array}{l}\text { 6. Reglas para uso de } \\
\text { celular en el colegio }\end{array}$ & - & - & -0.098 & -0.085 & $-0.107^{*}$ & 0.059 & $0.176^{* * *}$ & - \\
\hline 7. Nivel socioeconómico & 9.27 & 2.45 & $0.224^{* * *}$ & 0.082 & $0.251^{* * *}$ & $0.125^{* *}$ & $0.116^{*}$ & 0.006 \\
\hline
\end{tabular}


mayores puntajes en la mediación activa docente y de pares, y con el uso de la Internet en el colegio; se presentan mayores puntajes en los niveles socioeconómicos altos, además de mayores puntajes en comportamiento y exposición al riesgo. Precisamente el índice de riesgo sólo presenta correlación con las habilidades digitales.

La mediación activa docente se asocia positivamente con la mediación de los pares $(r=.48)$ y con el uso de la Internet en la institución educativa $(r=.23)$; la mediación con los pares también se asocia positivamente con el uso de la Internet en el colegio $(r=.23)$. Una mayor mediación docente $(r=.12)$ y de los pares $(r=.12)$ se correlaciona con un mayor nivel socioeconómico.

Por medio de un modelo de ecuación estructural se procedió a probar uno más para la predicción de las habilidades digitales y las actividades de riesgo, y para ello se tomaron como variables predictoras la mediación activa docente y de pares, el uso de Internet en la institución educativa, la presencia de reglas para el uso del teléfono celular y el nivel socioeconómico (Fig. 2). Se obtuvo un ajuste aceptable $(X 2=230.25 \mathrm{gl} 139 \mathrm{p}<.0001, \mathrm{CFI}=.94$, TLI $=.93$, RMSEA $=.05$, IC (90 por ciento) $=.04, .05$, $\mathrm{SRMR}=.06, \mathrm{GFI}=.98, \mathrm{AGFI}=.97)$.

Figura 4. Predictores asociados al contexto escolar de las habilidades digitales y la exposición a riesgos

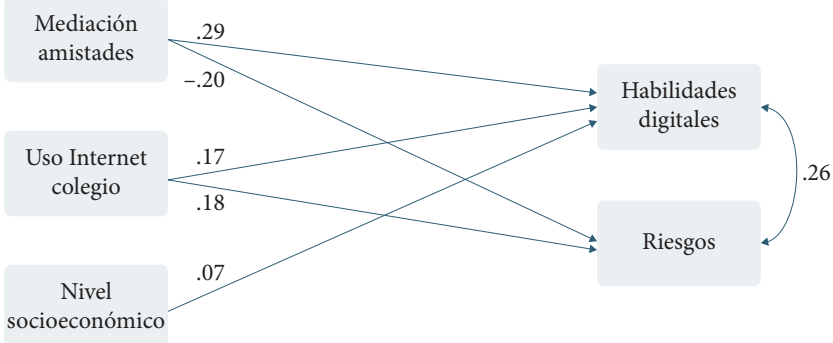

Fuente: elaboración propia.

Las habilidades digitales y el riesgo covarían positivamente, por lo que altos puntajes en las habilidades digitales se asocian a altos puntajes en actividades asociadas a riesgo $(\beta=.26, \mathrm{IC}=.11, .41, z=3.45, \mathrm{p}=.001)$.

Las habilidades digitales presentan una asociación positiva con el uso de la Internet en el colegio $(\beta=.17, \mathrm{IC}=.02, .36, z=2.18, \mathrm{p}=.03)$, la mediación activa de los pares $(\beta=.29, \mathrm{IC}=.13$, $.51, z=3.28, \mathrm{p}=.001)$ y la condición socioeconómica $(\beta=.07, \mathrm{IC}=.01, .13, z=2.45, \mathrm{p}=.01)$, de modo que un mayor uso de la Internet en el colegio, una mayor mediación activa de los pares y niveles socioeconómicos más altos predicen las habilidades digitales.

Los comportamientos y exposición a riesgos se asocian positivamente con el uso de Internet en el colegio $(\beta=.18, \mathrm{IC}=.02, .36, z=2.22$, $\mathrm{p}=.03$ ), y negativamente con la mediación acti- va de los pares $(\beta=-.20, \mathrm{IC}=-.39,-.02, z=-2.17$, $\mathrm{p}=.03$ ), de forma tal que un mayor uso de Internet en el colegio predice mayores comportamientos de riesgo, pero una mayor mediación activa de los pares predice una menor exposición y comportamiento de riesgo.

En lo que respecta al efecto mediador de la frecuencia de uso de Internet para actividades educativas en la asociación entre la mediación docente o de las amistades y las habilidades digitales o la presencia de riesgos, se encontraron resultados relevantes.

También se encontró que el uso de Internet en el colegio tiene un efecto de mediación parcial sobre la asociación positiva entre mediación activa docente y las habilidades digitales (efecto total: coef. .21, IC [ 95 por ciento] $=.07$, $.35, z=2.95, \mathrm{p}<.003$. Efecto directo: coef. .15, IC [95 por ciento] $=.01, .29, z=2.07, \mathrm{p}<.04$. Efecto 
indirecto: coef. .06, IC [95 por ciento] $=.02, .11$, $z=2.84, \mathrm{p}<.005)$. Así, la mediación docente se asocia positivamente con el uso de Internet en el colegio (coef. .39, IC [95 por ciento] $=.20$, $.58, z=4.10, \mathrm{p}<.001$ ), el uso de Internet en el colegio con las habilidades digitales (coef. .16, IC [ 95 por ciento] $=.08, .24, z=3.93, \mathrm{p}<.001)$ y la mediación docente con las habilidades digitales (coef. .15, IC [95 por ciento] $=.008, .29, z=2.07$, $\mathrm{p}<.009$ ). La mediación activa docente parece promover el uso de Internet en el colegio y esto es lo que facilita que la actividad docente tenga un efecto promotor de mayores habilidades digitales (Fig. 5).

No se halló un efecto mediador del uso de Internet en el colegio sobre la asociación positiva entre mediación activa docente y la presencia de riesgos en el uso de la red. Por otro lado, se encontró que el uso de Internet en el colegio tiene un efecto de mediación parcial sobre la asociación positiva entre mediación activa de las amistades y las habilidades digitales (efecto total: coef. .09, IC [ 95 por ciento] $=.06$, $.12, z=5.42, \mathrm{p}<.001$; efecto directo: coef. .07, IC [95 por ciento] $=.04, .11, z=4.52, \mathrm{p}<.001$; efecto indirecto: coef. .01, IC [ 95 por ciento] $=.001, .04$, $z=2.84, \mathrm{p}<.005)$. Así, la mediación de pares se asocia positivamente con el uso de Internet en el colegio (coef. .09, IC [ 95 por ciento] $=.02$, $.05, z=4.23, \mathrm{p}<.001)$; el uso de Internet en el colegio con las habilidades digitales (coef. .15, IC [95 por ciento] $=.07, .23, z=3.83, \mathrm{p}<.001)$; $\mathrm{y}$ la mediación de pares con las habilidades digitales (coef. .07, IC [ 95 por ciento] $=.04, .11, z=4.52$ $\mathrm{p}<.001$ ). La mediación activa de las amistades parece facilitar el uso de Internet en el colegio, y esto se asocia a un efecto promotor del aporte de las y los amigos sobre mayores habilidades digitales (Fig. 5).

Además, se encontró que el uso de Internet en el colegio tiene un efecto mediador sobre la asociación positiva entre mediación activa de las amistades y la presencia de riesgos (efecto total: coef. -.03 , IC [ 95 por ciento] $=-.09, .02, z=$ -1.15 , p .251; efecto directo: coef. -.06, IC [95 por ciento $]=-.12,-.001, z=-2.13, \mathrm{p}<.03$; efecto indirecto: coef. .03, IC [ 95 por ciento] $=.01, .05, z=3.13$, $\mathrm{p}<.002)$. Así, la mediación de las amistades se asocia positivamente con el uso de Internet en el colegio (coef. .10, IC [ 95 por ciento] $=.06, .14$, $z=4.86$, $\mathrm{p}<.001)$; el uso de Internet en el colegio con las habilidades digitales (coef. .30, IC [95 por ciento] $=.16, .45, z=4.09, \mathrm{p}<.001$ ); $y$ la mediación de las amistades con las habilidades digitales (coef. -.06 , IC [95 por ciento] $=-.12,-.001$, $z=-2.13, \mathrm{p}<.033)$. De esta manera, el efecto inhibidor de la mediación de las amistades sobre la presencia de riesgos en el uso de Internet está supeditado a la frecuencia de uso de dicha red en el colegio (Fig. 5).

Figura 5. Efectos mediadores encontrados de la frecuencia de uso de Internet en el colegio sobre las asociaciones entre los tipos de mediación estudiado y las habilidades digitales y la presencia de riesgos
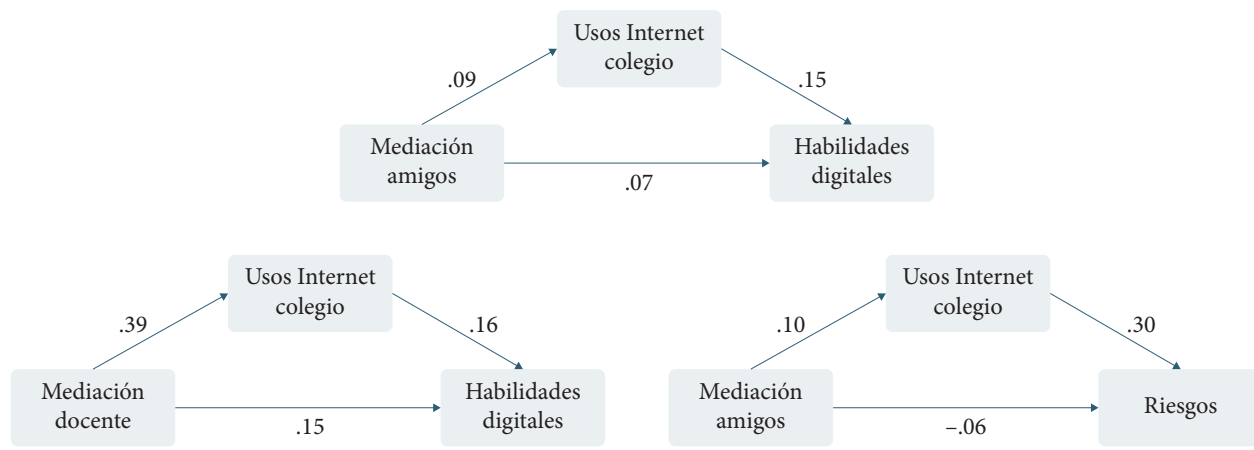

Fuente: elaboración propia. 
Asimismo, no se presentó un efecto moderador de la presencia de reglas de uso del teléfono celular sobre la asociación entre la mediación docente o de las amistades y las habilidades digitales o la presencia de riesgos.

\section{DISCUSIÓN}

Se encontró respaldo empírico a las hipótesis H2, H4, H7, H8, H9, H11. Un mayor uso de la Internet en el colegio, una mayor mediación activa de los pares y niveles socioeconómicos más altos se asocian a mayores habilidades digitales. En cuanto a los comportamientos y exposición a riesgos, un mayor uso de la Internet en el colegio se asocia a mayores comportamientos de riesgo, pero una mayor mediación activa de los pares se relaciona con una menor frecuencia de estos comportamientos.

Por otro lado, se encontró respaldo para las hipótesis H12, H14 y H15. El efecto promotor de la mediación activa docente sobre mayores habilidades digitales está mediado por la frecuencia de uso de Internet en el colegio. Así mismo, el efecto promotor de la mediación activa de las amistades sobre mayores habilidades digitales está mediado por la frecuencia de uso de Internet en el colegio. Además, el efecto inhibidor de la mediación de las amistades sobre la presencia de riesgos en el uso de esta red está supeditado a la frecuencia de su uso en el colegio.

No se encontró respaldo empírico para las hipótesis H16, H17, H18 y H19, ya que no se observaron evidencias de que la presencia de reglas sobre el uso de teléfonos celulares en el colegio juegue un papel relevante en la relación entre mediación docente y de pares con las habilidades digitales o conductas de riesgo.

Resulta de gran relevancia el hecho de que las y los docentes participantes en el estudio parecen no ejercer un efecto directo y evidente, ya sea protector o beneficioso, en el uso de la Internet, como se ha visto en otros contextos (Shin y Lwin, 2017; Berger y Wolling, 2019). Estos resultados pueden indicar que la mediación docente en el uso de Internet es muy limitada, posiblemente debido a las limitadas habilidades digitales para su uso pedagógico, de modo que no resultan ser fuente de información, guía, acompañamiento u orientación para ello. Estos resultados requieren de mayor investigación precisamente para indagar en específico en las habilidades digitales de las y los docentes, y la apropiación que se hace de las tecnologías digitales en el contexto de clase. Además, son un llamado de atención para fortalecer la guía, participación y acompañamiento docente en el uso de Internet en las instituciones de secundaria.

En esta línea, resulta interesante el papel mediador de la frecuencia de uso de Internet en el colegio en la relación entre mediación docente y habilidades digitales, el cual parece encontrarse relacionado con parte del modelo presentado por Pimdee y Leekitchwatana (2019); este estudio sostiene que las condiciones contextuales del colegio, así como las reglas de uso de Internet, la preparación de la institución hacia la conectividad y el trabajo de la institución en la formación docente son factores que influyen en los cambios conductuales de los docentes hacia la enseñanza del uso de esta red, ya que promueven una mayor utilización de los medios digitales en la preparación de las clases y una mayor evaluación de la utilidad de la Internet, lo que puede llevar a mejores habilidades digitales de los docentes $\mathrm{y}$, por ende, de las y los estudiantes.

Como se ha observado en investigaciones anteriores (Pérez y Torres, 2020), dentro del colegio la mediación docente se orienta más hacia la restricción del uso de Internet. Es posible que esto se deba, en parte, a las pobres competencias digitales de los mismos docentes además de una tendencia a la desconfianza y a evitar el riesgo. Lo anterior subraya la relevancia de analizar el papel de las habilidades digitales de los mismos docentes sobre el tipo de mediación que ejercen en las niñas, niños y adolescentes y, una vez más, el papel que juegan las instituciones de enseñanza primaria y secundaria en la formación de su personal docente 
en habilidades digitales, así como el establecimiento de reglas de uso de aparatos tecnológicos - como el teléfono celular-e Internet más flexibles u orientadas a usos educativos.

De igual forma sería relevante tomar en consideración la inclusión de programas educativos sobre los usos riesgosos de la Internet dentro del currículo académico, para que se pueda promover un mejor uso por parte de los adolescentes y fortalecer la mediación de los pares en los usos de la red. A esto podrían sumarse programas de educación para padres y madres en los cuales se pueda trabajar en la mejora de sus habilidades digitales y para formar una estructura de mediación activa más fuerte en la que tanto docentes como padres, madres y el grupo de pares puedan fungir como mediadores para la disminución de conductas de riesgo en línea.

El hecho de que un mayor uso de Internet en el contexto escolar se asocie a mayores habilidades digitales refiere, por un lado, al rol positivo que pueden ejercer las instituciones secundarias con estas características al promover sus beneficios (Berger y Wolling, 2019; Lorenz et al., 2016) y, por otro lado, a que el uso intensivo de Internet con objetivos educativos también parece promover una mayor exposición y comportamiento de riesgo, precisamente por la asociación entre habilidades digitales y riesgos cuando no existe una guía o acompañamiento claro de las y los docentes.

Ahora bien, en el contexto de los resultados descritos y comentados hasta ahora, la participación de las y los pares en el desarrollo de habilidades y en la reducción del riesgo es significativa. En consonancia con lo encontrado por Cerna et al. (2016), la mediación activa de las amistades resulta ser tanto un promotor de habilidades como un espacio protector contra posibles daños. En un contexto de ausencia de una guía clara por parte de las y los docentes, los pares despliegan una función fundamental.

En esta línea, es relevante destacar que esta relación se ve mediada por el uso de Internet en el colegio, lo cual puede implicar que el uso de reglas más flexibles y orientadas hacia un uso educativo y focalizado de la Internet puede ayudar a fortalecer el papel de los pares en el desarrollo de habilidades digitales y la reducción de riesgo en los/las adolescentes.

Otro aspecto que resulta particularmente interesante es el hecho de que la condición socioeconómica influye en las habilidades digitales, pero no en los comportamientos y exposición a riesgos. Sería relevante analizar si existe un rol moderador de la misma en la relación entre habilidades digitales y comportamiento de riesgo o si la presencia del riesgo es realmente independiente de la condición socioeconómica de los/las adolescentes; de esta forma se podrían diseñar programas diferenciados dependiendo de las condiciones socioeconómicas para fortalecer las habilidades digitales y prevenir comportamientos de riesgo de los menores de edad.

Para futuras investigaciones se requiere determinar el papel del tipo del colegio (público o privado) así como la zona geográfica (rural o urbana) en los resultados encontrados. Igualmente es importante conocer la asociación entre la mediación activa de las madres y los padres en interacción con la mediación docente y la de amistades, para determinar si el tipo de crianza tecnológica parental contribuye a entender el uso de Internet en el contexto educativo.

Finalmente, es necesario analizar con mayor detalle el uso del teléfono celular en la institución escolar con fines educativos y la asociación entre el empleo de dichos aparatos por parte de las y los docentes como herramienta educativa y el uso que hacen las y los estudiantes. Además, podría ser relevante investigar sobre el uso de software de plataformas educativas respecto del papel que puede tener el uso del teléfono celular como herramienta educativa y como mediador en la adquisición de habilidades digitales por parte de los menores de edad. 
BARbovschi, Mónica, Hana Macháčková y Kjartan Ólafsson (2015), "Underage Use of Social Network Sites: It's about friends", Cyberpsychology, Behavior, and Social Networking, vol. 18, núm. 6, pp. 328-332.

BArtau-Rojas, Isabel, Ana Aierbe-Barandiaran y Eider Oregui-González (2018), "Parental Mediation of the Internet use of Primary Students: Beliefs, strategies and difficulties", Comunicar, vol. 26, núm. 54, pp. 71-79.

Berger, Priscila y Jens Wolling (2019), “They Need More than Technology-Equipped Schools: Teachers' practice of fostering students' digital protective skills", Media and Communication, vol. 7, núm. 2, pp. 137-147.

Cerna, Alena, Hana Machackova y Lenka Dedkova (2016), "Whom to Trust: The role of mediation and perceived harm in support seeking by cyberbullying victims", Children \& Society, vol. 30, núm. 4, pp. 265-277.

ECCLES, Jacquelynne S. y Robert W. Roeser (2011), "Schools as Developmental Contexts During Adolescence", Journal of Research on Adolescence, vol. 21, núm. 1, pp. 225-241.

Global Kids Online (2021), Research Toolkit, en: http://globalkidsonline.net/tools/ (consulta: 11 de septiembre de 2021).

Helsper, Ellen J., Alexander J.A.M. van Deursen y Rebecca Eynon (2015,), "From Digital Skills to Tangible Outcomes: Full questionnaire", en: https://www.lse.ac.uk/media-and-communications/assets/documents/research/ projects/disto/From-Digital-Skills-to-Tangible-Outcomes-Full-Questionnaire..pdf (consulta: 11 de septiembre de 2021).

Helsper, Ellen y Alexander van Deursen (2018), "ICT Skills for the Future", en ITU (International Telecommunications Union) (ed.), Measuring the Information Society, Ginebra, ITU, pp. 21-50.

Hooper, Daire, Joseph Coughlan y Michael Mullen (2008), "Structural Equation Modelling: Guidelines for determining model fit", The Electronic Journal of Business Research Methods, vol. 6, núm. 1, pp. 53-60.

Hsu, Shinkuan (2015), "Exploring Teacher's Role in Students' Internet Overuse Problems: Instructional strategies and emotional support", Research of Educational Communications and Technology, vol. 112, pp. 1-18.

JiA, Jichao, Dongpin Li, Xiang Li, Yueyue Zhou, Yanhui Wang, Wenqiang Sun y Liyan Zhao (2018), "Peer Victimization and Adolescent Internet Addiction: The mediating role of psychological security and the moderating role of teacher-student relationships", Computers in Human Behavior, vol. 85, pp. 116-124.
Kalmus, Veronika y Triin Roosalu (2012), "Institutional Filters on Children's Internet Use: An additional explanation of cross-national differences in parental mediation", en Michel Walrave (ed.), E-youth: Balancing between opportunities and risks, Nueva York/Bruselas, Peter Lang, pp. 235-250.

Kalmus, Veronika, Cecilia von Feilitzen y Andra Siibak (2012), "Effectiveness of Teachers' and Peers' Mediation in Supporting Opportunities and Reducing Risks Online”, en Sonia Livingstone, Leslie Haddon y Anke Görzik (eds.), Children, Risk, and Safety on the Internet: Research and policy challenges in comparative perspective, Londres, Policy Press, pp. 245-256.

Kates, Aaron, Huang Wu y Chris Coryn (2018), “The Effects of Mobile Phone Use on Academic Performance: A meta-analysis", Computers \& Education, vol. 127, pp. 107-112.

LI, Dongping, Xian Li, Yanhui Wang, Liyan Zhao, Zhenzhou Bao y Fangfang Wen (2013), "School Connectedness and Problematic Internet Use in Adolescents: A moderated mediation model of deviant peer affiliation and self-control", Journal of Abnormal Child Psychology, vol. 41, pp. 1231-1242.

Livingstone, Sonia (2013), "Online Risk, Harm, and Vulnerability: Reflections on the evidence base for child Internet safety policy", ZER: Journal of Communication Studies, vol. 18, núm 35, pp. 13-28.

Livingstone, Sonia y Ellen Helsper (2010), "Balancing Opportunities and Risks in Teenagers' Use of the Internet: The role of online skills and Internet self-efficacy", New Media \& Society, vol. 12, núm. 2, pp. 309-329.

Livingstone, Sonia, Leslie Haddon, Anke Görzig y Kjartan Olafsson (2011), Risks and Safety on the Internet. The perspective of European children: Full findings and policy implications from the EU Kids Online survey of 9-16 year olds and their parents in 25 countries, Londres, EU Kids Online.

Livingstone, Sonia, Giovanna Mascheroni y Elizabeth Staksrud (2015), Developing a Framework for Researching Children's Online Risks and Opportunities in Europe, Londres, EU Kids Online, en: http://eprints.lse.ac.uk/64470/ (consulta: 11 de septiembre de 2021).

Livingstone, Sonia, Giovanna Mascheroni y Elizabeth Staksrud (2018), European Research on Children's Internet Use: Assessing the past and anticipating the future, New Media y Society, vol. 20, núm. 3, pp. 1103-1122.

Livingstone, Sonia, Dafna Lemish, Sun Sun Lim, Monica Bulger, Patricio Cabello, Magdalena Claro, Tania Cabello-Hut, Joe Khalil, Kristiina Kumpulainen, Usha S. Nayar, Priya 
Nayar, Jonghwi Park, Maria Melizza Tan, Jeanne Prinsloo y Bu Wei (2017), "Perspectives on Children's Digital Opportunities: An emerging research and policy agenda", Pediatrics, vol. 140, núm. S2, pp. S137-S141.

Lorenz, Ramona, Manuela Endberg y Birgit Eickelmann (2016), "Unterrichtliche Nutzung digitaler Medien von Lehrkräften der Sekundarstufe I in Deutschland und im Bundesländervergleich", en Wilfried Bos, Ramona Lorenz, Manuela Endberg, Birgit Eickelmann, Rudolf Kammerl y Stefan Welling (eds.), Schule Digital-Der Länderindikator 2016: Kompetenzen von Lehrpersonen der Sekundarstufe I im Umgang mit digitalen Medien im Bundesländervergleich, Münster, Waxmann, pp. 80-109.

O’Bannon, Blanche, Stewart Waters, Jennifer Lubke, J. Cady y KristinRearden (2017),“Teachers and Students Poised to Use Mobile Phones in the Classroom", Computers in the Schools, vol. 34, núm. 3, pp. 125-141.

PÉrez Sánchez, Rolando (2019), Informe Encuesta Kids Online Costa Rica, Universidad de Costa Rica/Fundación Paniamor.

PÉrez Sánchez, Rolando y David Torres Fernández (2020), "Mediación adulta de la Internet: un estudio cualitativo con adolescentes costarricenses", Revista Colombiana de Educación, vol. 1, núm. 79, pp. 39-60.

PimdeE, Paitoon y Punnee Leekitchwatana (2019), "Structural Equation Modeling of Teaching Behavior in Internet Use of Teachers in Thailand", TEM Journal, vol. 8, núm. 4, pp. 1499-1507.

Rodríguez de Dios, Isabel, Johanna van Oosten y Juan José Igartua (2018), "A Study of the Relationship between Parental Mediation and Adolescents' Digital Skills, Online Risks and Online Opportunities", Computers in $\mathrm{Hu}$ man Behavior, vol. 82, pp. 186-198.

SHin, Wonsun y May Lwin (2017), "How does 'Talking about the Internet with Others' Affect Teenagers' Experience of Online Risks? The role of active mediation by parents, peers, and school teachers", New Media \& Society, vol. 19, núm. 7, pp. 1109-1112.
SoH, Patrick Chin Hoi, Kok-Wai Chew, Klan Yeik Koay y Peng Hwa Ang (2018), “Parents vs Peers' Influence on Teenagers' Internet Addiction and Risky Online Activities", Telematics and Informatics, vol. 35, núm. 1, pp. 225-236.

Soo, Kadri, Veronika Kalmus y Mare Ainsaar (2015), "The Role of Estonian Teachers in the Social Mediation of Children's Internet Use", Estonian Journal of Education, vol. 3, núm. 2, pp. 156-185.

Sung, Yao Ting, Kuo-En Chang y Tzu Chien Liu (2016), "The Effects of Integrating Mobile Devices with Teaching and Learning on Students' Learning Performance: A meta-analysis and research synthesis", Computers \& Education, vol. 94, pp. 252-275.

Teneste Fiseha, Feven y Miriam Razon-Estrada (2018), "Internet Use Management Program: Its effects on the lifestyle of high school students", Catalyst, vol. 17, pp. 89-97.

Tingir, Seyfullah, Baki Cavlazoglu, Omer Caliskan, Onder Koklu y Seyma Intepe-Tingir (2017), "Effects of Mobile Devices on K-12 Students' Achievement: A meta-analysis", Journal of Computer Assisted Learning, vol. 33, núm. 4, pp. 355-369.

Van Deursen, Alexander, Cedric Courtois y Jan van Dijk (2014), "Internet Skills, Sources of Support, and Benefiting from Internet Use", International Journal of Human-Computer Interaction, vol. 30, pp. 278-290.

Van Deursen, Alexander y Jan van Dijk (2014), Digital Skills: Unlocking the information society, Nueva York, Palgrave-Macmillan.

WisharT, Jocelyn (2004), "Internet Safety in Emerging Educational Contexts", Computers \& Education, vol. 43, núm. 1-2, pp. 193-204. 